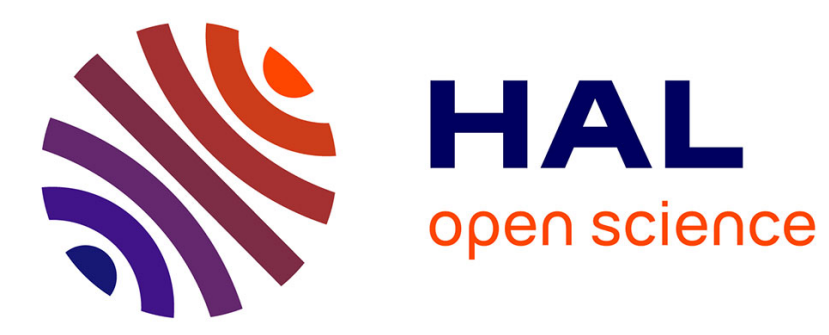

\title{
Effet du contre-ion et de l'électrolyte solubilisé sur des micelles ioniques
}

\author{
C. Cabos
}

\section{To cite this version:}

C. Cabos. Effet du contre-ion et de l'électrolyte solubilisé sur des micelles ioniques. Journal de Physique Lettres, 1983, 44 (24), pp.997-1006. 10.1051/jphyslet:019830044024099700 . jpa-00232300

\section{HAL Id: jpa-00232300 https://hal.science/jpa-00232300}

Submitted on 1 Jan 1983

HAL is a multi-disciplinary open access archive for the deposit and dissemination of scientific research documents, whether they are published or not. The documents may come from teaching and research institutions in France or abroad, or from public or private research centers.
L'archive ouverte pluridisciplinaire HAL, est destinée au dépôt et à la diffusion de documents scientifiques de niveau recherche, publiés ou non, émanant des établissements d'enseignement et de recherche français ou étrangers, des laboratoires publics ou privés. 
Classification

Physics Abstracts

$82.70 \mathrm{~K}-61.12$

\title{
Effet du contre-ion et de l'électrolyte solubilisé sur des micelles ioniques
}

\author{
C. Cabos \\ Groupe de Dynamique des Phases Condensées, \\ U.S.T.L., Place E. Bataillon, 34060 Montpellier Cedex, France
}

(Reçu le 4 juillet 1983, accepté le 27 octobre 1983)

\begin{abstract}
Résumé. - Les différences de taille et de forme de micelles ioniques de p-octylbenzènesulfonate de lithium et de sodium dans l'eau s'interprètent correctement à partir de la taille du contre-ion hydraté. L'effet de sel sur les micelles de p-octylbenzènesulfonate de sodium confirme ce rôle. L'étude est réalisée par diffusion des neutrons aux petits angles.

Abstract. - The differences in shape and size of ionic micelles of lithium and sodium p-octylbenzenesulfonate in water can be correctly interpreted by using the size of the hydrated counterion. The effect of salt upon micelles of sodium p-octylbenzenesulfonate is coherent with this rôle. This study can be achieved by small angle neutron scattering.
\end{abstract}

\section{Introduction.}

Après la diffusion centrale des rayons X (D.C.R.X.) [1] la technique de diffusion des neutrons aux petits angles (D.N.P.A.) - par la méthode du contraste variable [2] - a permis de déterminer les paramètres descriptifs de solutions micellaires ioniques directes en phase $\mathrm{L}_{1}$ (têtes polaires des molécules d'amphiphiles dirigées vers l'extérieur de la micelle) à condition d'étudier des solutions très diluées ( 2 à 3 fois la concentration micellaire critique).

Pour des concentrations plus élevées, les corrélations intermicellaires créent des interférences qui se superposent à la diffusion individuelle des particules. On peut alors décrire l'ordre local dans la phase micellaire et étudier sa variation en fonction de la concentration [3]. Ces corrélations se manifestent dans le profil de diffusion par la présence d'un anneau, caractéristique de la phase $\mathrm{L}_{1}$. En effet :

- On ne l'observe pas avec des solutions micellaires inverses (phase $L_{2}$ ) [4] ou non ioniques [5], dans le domaine des concentrations faibles ou moyennes (de l'ordre de $10^{-1}$ mole. $1^{-1}$ ).

- On peut le faire disparaître par adjonction de sels qui écrantent les interactions électrostatiques.

Il a paru intéressant de voir si, dans ce dernier cas, les micelles subissent des variations de taille et de forme. Nous avons limité cette étude à des solutions aqueuses de p-octylbenzènesulfonate de sodium $\left(\mathrm{C}_{8} \phi \cdot \mathrm{SO}_{3} \mathrm{Na}\right)$ dans lesquelles nous avons solubilisé successivement des chlorures alcalins : chlorures de lithium, de sodium et de césium. A titre de comparaison, nous avons déterminé les caractéristiques géométriques de p-octylbenzènesulfonate de lithium $\left(\mathrm{C}_{8} \phi \mathrm{SO}_{3} \mathrm{Li}\right)$ en phase $\mathrm{L}_{1}$. 
Pour l'essentiel, les expériences ont été réalisées par D.N.P.A. La figure 1 illustre la disparition progressive du maximum d'interaction par solubilisation d'un électrolyte $(\mathrm{LiCl})$. On a représenté sur ce diagramme l'intensité diffusée $I(q)$ (mesurée à une échelle arbitraire) en fonction du module du vecteur de diffusion $q, q=4 \pi \sin \theta / \lambda$ où $\lambda$ est la longueur d'onde du rayonnement et $2 \theta$ l'angle de diffusion.

L'amphiphile $\left(\mathrm{C}_{8}{ }^{\prime} \phi \mathrm{SO}_{3} \mathrm{Na}\right)$ a été solubilisé dans $\mathrm{D}_{2} \mathrm{O}$ afin d'obtenir un contraste élevé entre la particule et le solvant. La concentration $C$ en amphiphile est maintenue constante et égale à 0,1 mole $.1^{-1}$ pour toutes les solutions. En l'absence de sel, la position $q_{\mathrm{M}}$ du maximum observé $\left({ }^{1}\right)$ donne, en première approximation, la distance intermicellaire moyenne. Pour des concentrations croissantes, le déplacement de ce maximum vers les grandes valeurs de $q$ est qualitativement significatif d'une diminution de la distance intermicellaire [3].

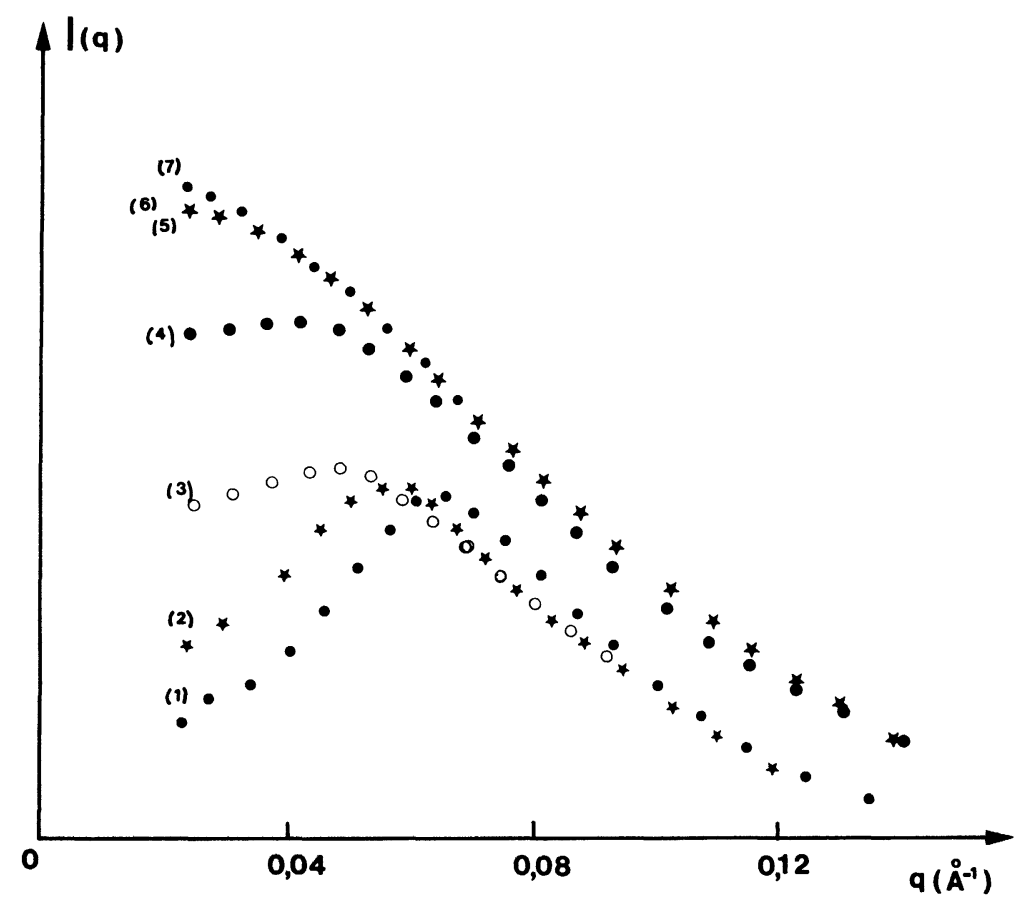

Fig. 1. - Diagramme de diffusion, à une échelle relative, de solutions micellaires de $\mathrm{C}_{8} \phi \mathrm{SO}_{3} \mathrm{Na}$ $\left(c=0,1\right.$ mole. $\left.1^{-1}\right)$ pour différentes concentrations en sel (en mole. $\left.1^{-1}\right)$. (1) $\left[\mathrm{Li}^{+}\right]=0,(2)\left[\mathrm{Li}^{+}\right]=0,005$, (3) $\left[\mathrm{Li}^{+}\right]=0,01$, (4) $\left[\mathrm{Li}^{+}\right]=0,02,(5)\left[\mathrm{Li}^{+}\right]=0,03,(6)\left[\mathrm{Li}^{+}\right]=0,04$, (7) $\left[\mathrm{Li}^{+}\right]=0,05$. Les courbes (4) $\rightarrow$ (7) ont été décalées vers le haut.

[Relative intensities of micellar solutions of $\mathrm{C}_{8} \phi \mathrm{SO}_{3} \mathrm{Na}\left(c=0.1\right.$ mole. $\left.1^{-1}\right)$ for different salt concentrations (in mole. $1^{-1}$ ). (1) $\left[\mathrm{Li}^{+}\right]=0$, (2) $\left[\mathrm{Li}^{+}\right]=0.005$, (3) $\left[\mathrm{Li}^{+}\right]=0.01,(4)\left[\mathrm{Li}^{+}\right]=0.02,(5)\left[\mathrm{Li}^{+}\right]=0.03$, (6) $\left[\mathrm{Li}^{+}\right]=0.04,(7)\left[\mathrm{Li}^{+}\right]=0.05$. Curves $(4) \rightarrow(7)$ have been shifted upwards. $]$

(1) L'observation d'un maximum, sur la courbe de diffusion relative à une solution diluée, peut dans certains cas être reliée à la structure de la particule diffusante. Le carré du facteur de structure $I_{1}(q)$ d'une particule sphérique à deux niveaux de densité $\rho_{1}$ (au centre) et $\rho_{2}$ dans un solvant $\rho_{\mathrm{S}}$ présente un maximum si $\rho_{1}<\rho_{2}$ et $\rho_{2}>\rho_{\mathrm{S}}$ [7]. Le choix de $\mathrm{D}_{2} \mathrm{O}$ comme solvant conduit ici à $\rho_{1}<\rho_{2}<\rho_{3}$. On peut alors vérifier que $I_{1}(q)$ est une fonction monotone décroissante quel que soit le modèle micellaire retenu. 
Une faible addition de sel $\left(\left[\mathrm{Li}^{+}\right]=10^{-2}\right.$ mole..$\left.^{-1}\right)$ est suffisante pour que le maximum d'interaction observé précédemment disparaisse en partie sans que la diffusion au-delà de $q_{\mathrm{M}}$ soit sensiblement modifiée. Au cours de cette atténuation du pic, $q_{\mathrm{M}}$ se déplace légèrement vers les petites valeurs de $q$. Une augmentation de la concentration en sel entraîne une disparition progressive du maximum qui est totale pour $\left[\mathrm{Li}^{+}\right]=5 \times 10^{-2}$ mole. $1^{-1}$. On peut alors considérer que les phénomènes d'interactions sont totalement écrantés.

\section{Rappels théoriques et hypothèses [2].}

Soit $\rho(r)$ la fonction décrivant la variation de la densité de longueur de diffusion cohérente d'une particule dans un solvant dont la densité uniforme est $\rho_{\mathrm{s}}$. On peut schématiquement représenter $\rho(r)$ par une fonction créneau : figure 2.

Le coeur micellaire de volume $v_{\text {par }}$ regroupe les chaines paraffiniques hydrophobes de densité $\rho_{\mathrm{par}}$. Dans l'hypothèse, couramment admise [6], d'une structure désordonnée, quasi liquide des chaînes, on peut calculer $\rho_{\text {par }}$. Nous admettrons par ailleurs que $\rho_{\text {par }}$ a la même valeur dans les micelles de $\mathrm{C}_{8} \phi \mathrm{SO}_{3} \mathrm{Na}$ et de $\mathrm{C}_{8} \phi \mathrm{SO}_{3} \mathrm{Li}$ quel que soit l'électrolyte solubilisé.

La partie polaire de la micelle, de volume $v_{\text {pol }}$, regroupe dans cette étude les cycles benzéniques, les ions sulfonates et un certain nombre de contre-ions qui peuvent être considérés comme liés à la particule. Nous désignerons par $\rho_{\text {pol }}$ la densité de longueur de diffusion cohérente de cette partie de la micelle. $\rho_{\mathrm{pol}}$ n'est pas connu a priori.

Soit $\langle\rho\rangle$ la densité moyenne de la particule de volume $v$ ( $v$ représente le volume exclu au solvant). Alors :

$$
\langle\rho\rangle=x \rho_{\mathrm{par}}+(1-x) \rho_{\mathrm{pol}}
$$

où $x=v_{\mathrm{par}} / v$.

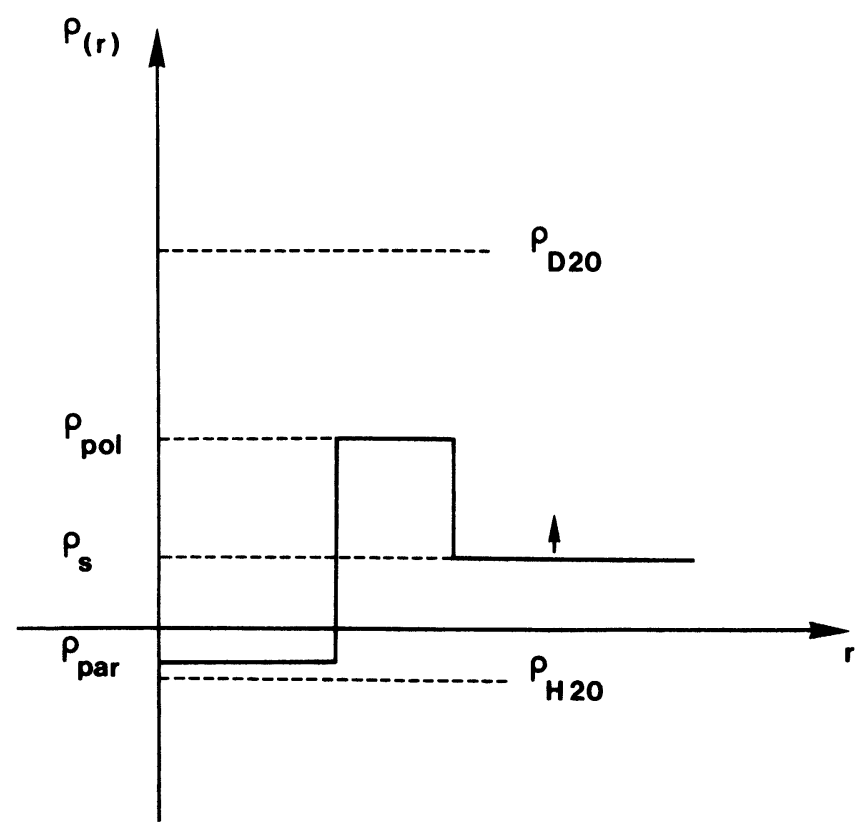

Fig. 2. - Représentation schématique de la fonction de répartition $\rho(r)$ de la densité de longueur de diffusion cohérente dans la micelle. La flèche indique le sens de variation de la densité $\rho_{\mathrm{S}}$ du solvant lorsque le rapport $\mathrm{D}_{2} \mathrm{O} / \mathrm{H}_{2} \mathrm{O}$ augmente.

[Schematic representation of the repartition function $\rho(r)$ of the density of scattering length in the micelles. The arrow shows the direction of the solvent density $\rho_{\mathrm{S}}$ when the ratio $\mathrm{D}_{2} \mathrm{O} / \mathrm{H}_{2} \mathrm{O}$ increases.] 
2. 1 Diffusion À L'ANGLe NUl. - Pour une particule isolée, la racine carrée de l'intensité diffusée à l'angle nul est une fonction linéaire de la densité de longueur de diffusion du solvant :

$$
\left[i\left(0, \rho_{\mathrm{S}}\right)\right]^{1 / 2}=\left(\langle\rho\rangle-\rho_{\mathrm{S}}\right) v .
$$

La possibilité, en D.N.P.A., de faire varier $\rho_{S}$ (en utilisant des mélanges $\mathrm{H}_{2} \mathrm{O} / \mathrm{D}_{2} \mathrm{O}$ ) permet donc, sans hypothèses supplémentaires (de forme notamment) de déterminer $\langle\rho\rangle$ et $v$ par calibration des mesures d'intensité [2]. Le nombre $n$ de monomères par micelle s'en déduit ainsi que $v_{\text {par }}$ :

$$
v_{\text {par }}=n \psi_{\text {par }}
$$

où $\psi_{\text {par }}$ est le volume d'une chaîne paraffinique.

La relation 1 conduit alors à la détermination de $x$ et de $\rho_{\text {pol }}$.

2.2 RAYON DE GIRATION. - Dans la région des petites valeurs de $q$, l'intensité diffusée peut être correctement représentée par l'approximation de Guinier [7] :

$$
i(q)=i(0) \exp \left[-q^{2} R_{\mathrm{G}}^{2} / 3\right]
$$

où $R_{\mathrm{G}}$ représentant le " rayon de giration " de la particule est défini par

$$
R_{\mathrm{G}}^{2}=\frac{\rho(r) r^{2} \mathrm{~d} v}{\rho(r) \mathrm{d} v}
$$

2.3 DiffUSiON AUX GRANDES VAlEURS $q$. - A l'autre extrémité de la courbe de diffusion, l'existence d'une limite asymptotique de l'intensité diffusée peut être reliée aux surfaces de séparation $S_{i j}$ entre les milieux de densité $\rho_{i}$ et $\rho_{j}$ suivant la loi de Porod [8] :

$$
(\lim q \rightarrow \infty) q^{4} i(q)=2 \pi \sum\left(\rho_{i}-\rho_{j}\right)^{2} S_{i j} .
$$

Remarquons que les relations $1,2,3$ et 4 n'ont d'intérêt pratique que si les micelles présentent, dans un domaine limité de concentration, une faible polydispersité en taille, ce que nous admettrons [3].

\section{Etude expérimentale.}

3.1 Appareillage. - Cette étude a été réalisée sur le montage expérimental de diffusion des neutrons - D11 - installé sur le réacteur à haut flux de l'Institut Laue-Langevin à Grenoble, dans les conditions expérimentales suivantes : $\lambda=4,2 \AA$, distance échantillon-détecteur $L=5,53 \mathrm{~m}$. Le domaine en $q$ ainsi défini $\left(1,6 \times 10^{-2} \AA^{-1}<q<8,6 \times 10^{-2} \AA^{-1}\right)$ vérifie la condition de Guinier [7] $\left(q R_{\mathrm{G}}<1\right)$ pour des rayons de giration inférieurs à $20 \AA$. Toutes les mesures ont été faites à $40^{\circ} \mathrm{C}\left( \pm 1^{\circ} \mathrm{C}\right)$. La cellule utilisée est une cuve plane en quartz d'épaisseur $1 \mathrm{~mm}$.

3. 2 Solutions. - Les détergents $\mathrm{C}_{8} \phi \mathrm{SO}_{3} \mathrm{Na}$ et $\mathrm{C}_{8} \phi \mathrm{SO}_{3} \mathrm{Li}$ ont été synthétisés au laboratoire en utilisant la méthode proposée par Gray [9]. Les chlorures alcalins sont des produits Merck suprapurs. Les propriétés physicochimiques des solutions aqueuses de $\mathrm{C}_{8} \phi \mathrm{SO}_{3} \mathrm{Na}$ en présence de sel, ont été étudiées par diverses méthodes [10] : solubilité, viscosité, autodiffusion. Nous retiendrons de cette étude les points suivants :

- L'adjonction de $\mathrm{NaCl}$ ne modifie pas la solubilité de $\mathrm{C}_{8} \phi \mathrm{SO}_{3} \mathrm{Na}$. Par contre, celle-ci augmente sensiblement en présence de $\mathrm{LiCl}$ ou de $\mathrm{CsCl}$.

- La concentration micellaire critique (c.m.c.) du détergent a pu être déterminée par autodiffusion du contre-ion. Sa valeur diminue en fonction de la concentration en sel ajouté suivant la relation de Shinoda [11]. A $40^{\circ} \mathrm{C}$, la c.m.c. pour des solutions de $\mathrm{C}_{8} \phi \mathrm{SO}_{3} \mathrm{Na}$ et $\mathrm{C}_{8} \phi \mathrm{SO}_{3} \mathrm{Li}$ 
est respectivement égale à $1,24 \times 10^{-2}$ mole. $1^{-1}$ et $1,34 \times 10^{-2}$ mole. $1^{-1}$. Pour une concentration en sel ajouté $(\mathrm{NaCl})$ de $5 \times 10^{-2}$ mole. $1^{-1}$, la c.m.c. de $\mathrm{C}_{8} \phi \mathrm{SO}_{3} \mathrm{Na}$ est égale à 0,8 mole $1^{-1}$.

Les volumes spécifiques $\bar{v}$ des amphiphiles ont été mesurés à l'aide d'un densitomètre de Kratky. La variation relative de $\bar{v}$ en fonction du sel ajouté est de l'ordre de $10^{-3}$. Elle est négligeable en première approximation. A $40^{\circ} \mathrm{C}, \bar{v}\left(\mathrm{C}_{8} \phi \mathrm{SO}_{3} \mathrm{Na}\right)=0,803 \mathrm{~cm}^{3} \cdot \mathrm{g}^{-1}$. Les longueurs de diffusion cohérente utilisées sont les suivantes : $\rho_{\mathrm{par}}=-0,54 \times 10^{10} \mathrm{~cm}^{-2} \cdot \rho_{\mathrm{S}}$ dépend du mélange $\mathrm{H}_{2} \mathrm{O} / \mathrm{D}_{2} \mathrm{O}$ qui est réalisé par pesée et peut varier de $-0,57 \times 10^{10} \mathrm{~cm}^{-2}\left(\rho_{\mathrm{S}}=\rho_{\mathrm{H}_{2} \mathrm{O}}\right)$ à $6,4 \times 10^{10} \mathrm{~cm}^{-2}\left(\rho_{\mathrm{S}}=\rho_{\mathrm{D}_{2} \mathrm{O}}\right)[3]$.

3.3 Mesure De LA Diffusion. - La valeur extrapolée de l'intensité à l'angle nul doit théoriquement être déterminée pour une dilution infinie [2] c'est-à-dire pratiquement pour $c \rightarrow$ c.m.c. Il est donc nécessaire de mesurer $I(q)$ pour plusieurs concentrations. En fait, deux solutions seulement de concentrations différentes ont pu être étudiées dans chaque cas. Il n'a pas été possible - pour des raisons de temps d'expérience - de réaliser davantage de mesures. Néanmoins, on a pu vérifier que $I(q \rightarrow 0) / c$ reste constant aux incertitudes près pour les deux valeurs de la concentration en amphiphiles qui ont été retenues : $c_{1}=0,05$ mole $1^{-1}$ et $c_{2}=0,1$ mole. $1^{-1}$. Pour chacune de ces concentrations, la concentration en sel est celle qui écrante les phénomènes d'interaction. Celle-ci est suffisamment faible pour qu'on puisse, en première approximation conserver le schéma d'interprétation qui a été donné. Ceci revient à considérer la solution comme un mélange binaire eau-détergent [17].

\section{Résultats expérimentaux et interprétation.}

On a pu vérifier par D.C.R.X. que l'intensité diffusée, mesurée à une échelle relative, aux grandes valeurs de $q\left(q>2 \times 10^{-1} \AA^{-1}\right)$ obéit à la loi de Porod. Ceci confirme l'hypothèse faite précédemment concernant $\rho(r)$ : le système étudié est formé de la juxtaposition de milieux de densités électroniques différentes avec des discontinuités du premier ordre [12]. L'équation 4 permet d'autre part de déterminer $S_{i j}$ dès lors que l'intensité est connue à l'échelle absolue. Cependant, la faiblesse du signal mesuré dans cette partie du diagramme ne permet pas d'envisager une exploitation quantitative précise de la loi aussi bien en D.N.P.A. qu'en D.C.R.X.

La figure 3 montre la variation linéaire de $\left[I\left(0, \rho_{\mathrm{S}}\right) / c\right]^{1 / 2}$ en fonction de la densité de longueur de diffusion du solvant. L'intersection des droites représentées avec l'axe des abscisses donne la valeur de $\langle\rho\rangle$ pour chacune des solutions micellaires. Notons que la droite relative à la solution $\left[\mathrm{C}_{8} \phi \mathrm{SO}_{3} \mathrm{Na}+\mathrm{NaCl}\right]$ très voisine de la droite (a) n'a pas été représentée sur la figure 3 . Connaissant le volume exclu $v$ d'une micelle de $\mathrm{C}_{8} \phi \mathrm{SO}_{3} \mathrm{Na}$ [2], on peut dresser un tableau de résultats relatifs aux systèmes étudiés : tableau I. La précision des résultats est de l'ordre de $5 \%$ pour $\langle\rho\rangle$ et $v$ et de $10 \%$ pour.les autres paramètres.

La détermination de $\rho_{\mathrm{pol}}$ (Tableau I) permet de préparer des solutions dans lesquelles $\rho_{\mathrm{S}}=\rho_{\mathrm{pol}}$ (Fig. 2). Dans ces conditions, il est clair que le rayon de giration mesuré sur le diagramme de Guinier (Fig. 3) est celui de la partie paraffinique de la micelle; il est noté $\left(R_{\mathrm{G}}\right.$ par) et peut être relié sans ambiguïté aux paramètres géométriques d'un modèle simple, sphère ou ellipsoïde, de volume $v_{\mathrm{m}}$. De la comparaison de $v_{\mathrm{m}}$ avec $v_{\text {par }}$ (déterminé sans hypothèse de forme), on peut déduire le modèle le plus probable relatif à la forme de la particule (Tableau II).

La surface disponible par tête polaire $\left(S_{\text {pol }}\right)$ à l'interface hydrophobe-hydrophile peut être calculée en tenant compte de l'encombrement du cycle benzénique rattaché à la chaîne paraffinique.

4.1 MiCelles DiReCTeS DE $\mathrm{C}_{8} \phi \mathrm{SO}_{3} \mathrm{Li}$. - Le volume exclu d'une micelle de $\mathrm{C}_{8} \phi \mathrm{SO}_{3} \mathrm{Li}$ est nettement inférieur à celui d'une micelle de $\mathrm{C}_{8} \phi \mathrm{SO}_{3} \mathrm{Na}$. Il est clair que le contre-ion joue un rôle essentiel dans la taille de la particule. Or, on sait que le rayon ionique du lithium est inférieur à celui du sodium $\left(r_{\left(\mathrm{Li}^{+}\right)}=0,68 \AA, r_{\left(\mathrm{Na}^{+}\right)}=0,97 \AA\right)$ mais que l'hydratation de l'ion $\mathrm{Li}^{+}$ 


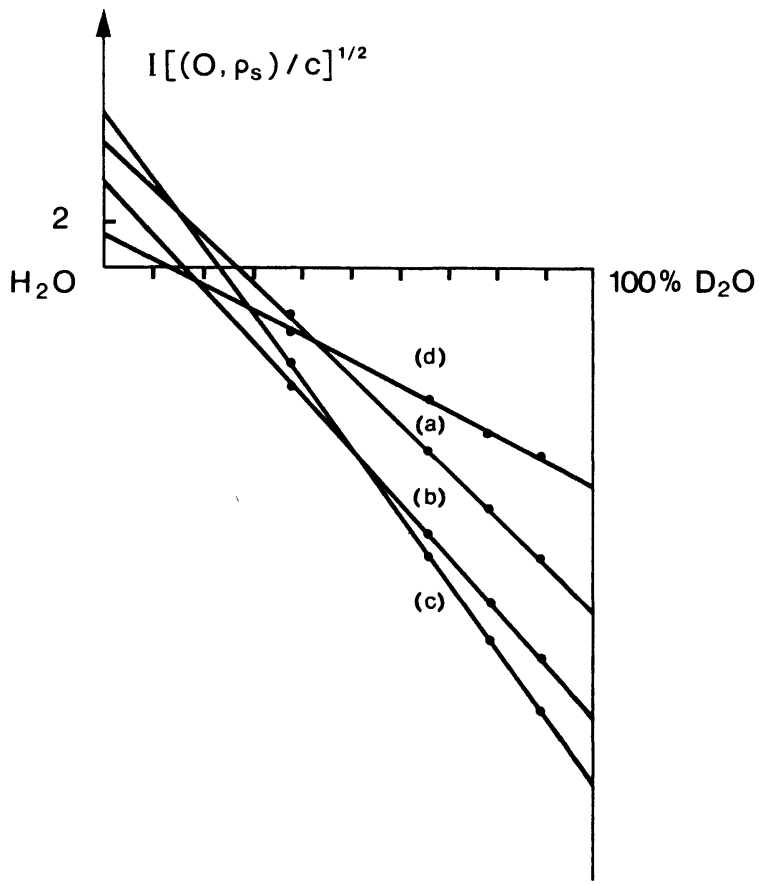

Fig. 3. - La racine carrée de $I(0) / c$ (à une échelle arbitraire) est une fonction linéaire de la densité de longueur de diffusion du solvant : $a$ ) micelles $\left.\mathrm{C}_{8} \phi \mathrm{SO}_{3} \mathrm{Na}, b\right)$ micelles $\mathrm{C}_{8} \phi \mathrm{SO}_{3} \mathrm{Na}+\mathrm{LiCl}, c$ ) micelles $\left.\mathrm{C}_{8} \phi \mathrm{SO}_{3} \mathrm{Na}+\mathrm{CsCl}, d\right)$ micelles $\mathrm{C}_{8} \phi \mathrm{SO}_{3} \mathrm{Li}$.

[The square root of $I(0) / c$ (in arbitrary units) is a linear function of the scattering length density of solvent : a) micelles $\left.\mathrm{C}_{8} \dot{\phi} \mathrm{SO}_{3} \mathrm{Na}, b\right)$ micelles $\mathrm{C}_{8} \phi \mathrm{SO}_{3} \mathrm{Na}+\mathrm{LiCl}, c$ ) micelles $\mathrm{C}_{8} \phi \mathrm{SO}_{3} \mathrm{Na}+\mathrm{CsCl}, d$ ) micelles $\mathrm{C}_{8} \phi \mathrm{SO}_{3} \mathrm{Li}$.]

Tableau I. - Résultats expérimentaux.

[Experimental results.]

\begin{tabular}{|c|c|c|c|c|c|c|}
\hline $\begin{array}{c}\text { Solutions } \\
\text { micellaires }\end{array}$ & $\begin{array}{c}\langle\rho\rangle \\
10^{10} \mathrm{~cm}^{-2}\end{array}$ & $\begin{array}{c}v \\
\left(\AA^{3}\right)\end{array}$ & $n$ & $\begin{array}{c}v_{\text {par }} \\
\left(\AA^{3}\right)\end{array}$ & $\begin{array}{c}\rho_{\text {pol }} \\
\mathrm{cm}^{-2}\end{array}$ & $x=\frac{v_{\text {par }}}{v}$ \\
\hline $\mathrm{C}_{8} \phi \mathrm{SO}_{3} \mathrm{Na}$ & 1,25 & 37500 & 80 & 19000 & 3,2 & 0,51 \\
\hline $\begin{array}{c}\mathrm{C}_{8} \phi \mathrm{SO}_{3} \mathrm{Na} \\
+\mathrm{NaCl}\end{array}$ & 1,25 & 37000 & 78 & 19000 & 3,2 & 0,52 \\
\hline $\begin{array}{c}\mathrm{C}_{8} \phi \mathrm{SO}_{3} \mathrm{Na} \\
+\mathrm{LiCl}\end{array}$ & 0,80 & 40000 & 85 & 21000 & 2,2 & 0,52 \\
\hline $\begin{array}{c}\mathrm{C}_{8} \phi \mathrm{SO}_{3} \mathrm{Na} \\
+\mathrm{CsCl}\end{array}$ & 1,15 & 54000 & 115 & 28000 & 3,0 & 0,52 \\
\hline $\mathrm{C}_{8} \phi \mathrm{SO}_{3} \mathrm{Li}$ & 0,60 & 23000 & 50 & 12500 & 1,9 & 0,53 \\
\hline
\end{tabular}


Tableau II. - Modèles et dimensions micellaires.

[Models and micellar sizes.]

\begin{tabular}{|c|c|c|c|c|c|c|}
\hline $\begin{array}{c}\text { Solutions } \\
\text { micellaires }\end{array}$ & $\begin{array}{c}\left(R_{\mathrm{G}} \mathrm{par}\right) \\
\pm 0,4 \AA\end{array}$ & $e$ & $\begin{array}{c}a \\
(\AA)\end{array}$ & $\begin{array}{c}b \\
(\AA)\end{array}$ & $\begin{array}{c}v_{\mathrm{m}} \\
\left(\AA^{3}\right)\end{array}$ & $\begin{array}{c}S_{\mathrm{pol}} \\
\left(\AA^{2}\right)\end{array}$ \\
\hline $\mathrm{C}_{8} \phi \mathrm{SO}_{3} \mathrm{Li}$ & 11,3 & $\begin{array}{c}1 \\
(\text { sphère })\end{array}$ & 14,6 & 14,6 & 13000 & 90 \\
\hline $\mathrm{C}_{8} \phi \mathrm{SO}_{3} \mathrm{Na}$ & 12,8 & 1,35 & 19,7 & 14,6 & 18000 & 70 \\
\hline $\begin{array}{c}\mathrm{C}_{8} \phi \mathrm{SO}_{3} \mathrm{Na} \\
+\mathrm{NaCl}\end{array}$ & 12,6 & 1,3 & 19,7 & 14,6 & 18000 & 70 \\
\hline $\begin{array}{c}\mathrm{C}_{8} \phi \mathrm{SO}_{3} \mathrm{Na} \\
+\mathrm{LiCl}\end{array}$ & 13,5 & 1,5 & 21,9 & 14,6 & 19500 & 70 \\
\hline $\begin{array}{c}\mathrm{C}_{8} \phi \mathrm{SO}_{3} \mathrm{Na} \\
+\mathrm{CsCl}\end{array}$ & 15,5 & 1,8 & 27,5 & 15,3 & 27000 & 60 \\
\hline
\end{tabular}

est supérieure à celle de l'ion $\mathrm{Na}^{+}$'[13]. Dans une première couche, 6 molécules d'eau sont liées à $\mathrm{Li}^{+}$et 5 pour $\mathrm{Na}^{+}$; dans une seconde couche, l'écart augmente : 7 et 3,5 molécules d'eau respectivement pour $\mathrm{Li}^{+}$et $\mathrm{Na}^{+}$. L'ion $\mathrm{Li}^{+}$, fortement hydraté, plus volumineux que l'ion $\mathrm{Na}^{+}$, neutralise mal la charge micellaire, ce qui favorise la répulsion électrostatique des têtes polaires. La surface spécifique $S_{\text {pol }}$ augmente, ce qui impose une diminution du nombre de monomères par micelle. On constate, par ailleurs, une diminution de $\langle\rho\rangle$ (par rapport à $\mathrm{C}_{8} \phi \mathrm{SO}_{3} \mathrm{Na}$ ) due à l'influence des ions $\mathrm{Li}^{+}$liés à la micelle et dont la longueur de diffusion cohérente est négative [14].

La comparaison de $v_{\text {par }}$ avec $v_{\mathrm{m}}$ montre qu'un modèle sphérique est ici satisfaisant. En effet, pour $\left(R_{\mathrm{G}}\right.$ par $)=11,3 \AA, v_{\mathrm{m}}=\frac{4}{3} \pi R_{\mathrm{par}}^{3}$ avec $R_{\mathrm{par}}^{2}=\frac{5}{3}\left(R_{\mathrm{G}}^{2}\right.$ par $)$ soit $v_{\mathrm{m}} \simeq 13000 \AA^{3}$. La surface disponible par tête polaire, calculée pour ce modèle, confirme l'augmentation de $S_{\text {pol }}$ (par rapport à $\left.\mathrm{C}_{8} \phi \mathrm{SO}_{3} \mathrm{Na}\right)$.

4.2 Micelles DireCtes DE $\mathrm{C}_{8} \phi \mathrm{SO}_{3} \mathrm{Na}$ en L'ABSENCE DE SEL. - Le rayon de giration de la partie paraffinique $\left(\left(R_{\mathrm{G}}\right.\right.$ par $\left.)=12,8 \AA\right)$ conduit, dans l'hypothèse d'un modèle sphérique, à $R_{\text {par }}=16,5 \AA$ valeur bien supérieure à la longueur de la chaîne paraffinique étirée $\left(l_{\text {par }}\right)$ de la molécule de $\mathrm{C}_{8} \phi \mathrm{SO}_{3} \mathrm{Na}$. $\left(l_{\text {par }}\right)$ peut être calculé à partir d'un modèle moléculaire [15] suivant la relation $\left(l_{\mathrm{par}}\right)=1,5+1,265 n_{\mathrm{c}}$ où $n_{\mathrm{c}}$ est le nombre d'atomes de carbone de la chaîne, soit $\left(l_{\text {par }}\right) \simeq 12 \AA$.

Si l'on veut conserver le modèle sphérique, il faut faire l'hypothèse de l'existence d'un trou dans le coeur micellaire dont le volume serait de l'ordre de $\frac{4}{3} \pi a^{3}-400 \AA^{3}\left(a=R_{\mathrm{par}} \simeq l_{\mathrm{par}}\right)$. Expérimentalement, il n'est pas possible de déceler un tel volume dont la valeur est inférieure à l'incertitude absolue commise dans l'évaluation de $v_{\mathrm{par}}$. Par ailleurs, il n'existe pas d'arguments en faveur de cette hypothèse.

Un autre modèle, suggéré par Tanford [15], rend mieux compte des résultats expérimentaux : c'est l'ellipsoïde de révolution de demi-axes $(a, b, b)$ dans lequel $b \simeq l_{\text {par }}$. Il est délicat de donner ici, de façon rigoureuse, les dimensions de ce modèle compte tenu des incertitudes qui affectent les résultats bruts. Néanmoins, un certain nombre de points peuvent être dégagés. 
Cet ellipsoïde est de type "allongé » (allongement $e=a / b>1$ ). En effet, pour un volume paraffinique donné s'exprimant suivant la relation $v_{\mathrm{m}}=\frac{4}{3} \pi e b^{3}, e>1$ si $b<R_{\mathrm{par}}$. Par ailleurs, $e, b$ et $R_{\mathrm{G}}$ sont liés par la relation $R_{\mathrm{G}}=b\left(\frac{2+e^{2}}{5}\right)^{1 / 2}$.

Dans le cas des micelles de $\mathrm{C}_{8} \phi \mathrm{SO}_{3} \mathrm{Na}$, les paramètres géométriques sont indiqués dans le tableau II. On peut constater que $b$ est, aux incertitudes près, identique au rayon paraffinique de la micelle sphérique de $\mathrm{C}_{8} \phi \mathrm{SO}_{3} \mathrm{Li}$. Cependant $b$ reste supérieur à $\left(l_{\text {par }}\right)$, ce qui peut s'interpréter par un décalage radial des chaînes pouvant donner un aspect plissé à l'interface.

L'allongement de cet ellipsoïde est suffisamment faible pour que la particule puisse toujours être considérée comme globulaire et pratiquement la surface disponible par tête polaire $\left(S_{\text {pol }}\right)$ est la même que pour le modèle sphérique.

4.3 MiCelles DiReCTES DE $\mathrm{C}_{8} \phi \mathrm{SO}_{3} \mathrm{Na}$ EN PRÉSENCE DE SEL. - L'adjonction de $\mathrm{NaCl}$ à une solution de $\mathrm{C}_{8} \phi \mathrm{SO}_{3} \mathrm{Na}$ entraîne une disparition progressive du maximum d'interaction comparable à celle qui est observée sur la figure 1 . Le calcul des principaux paramètres descriptifs de la micelle s'effectue donc ici dans de bonnes conditions contrairement à une détermination précédente [1]. Les résultats expérimentaux obtenus indiquent qu'il n'y a pas d'évolution notable de la forme et de la taille des particules. L'électrolyte ne joue ici qu'un rôle d'écran. Il n'en est plus de même lorsque le cation solubilisé est différent du cation provenant de l'amphiphile.

- C'est avec la solubilisation d'ions $\mathrm{Cs}^{+}$qu'une modification sensible des diagrammes de diffusion peut être observée (Figs. 2 et 3 ). Le gonflement de la micelle est ici très net (de l'ordre de $50 \%)$. Les mesures de $v_{\text {par }}$ et de $\left(R_{\mathrm{G}}\right.$ par) ne sont compatibles qu'avec un accroissement de l'anisotropie. Le modèle précédent d'ellipsoïde allongée peut toutefois être conservé avec une croissance de $b$ et un allongement voisin de 1,8. Corrélativement, la surface disponible par tête polaire diminue légèrement. Ces modifications structurales peuvent s'interpréter par le rôle stérique de l'ion $\mathrm{Cs}^{+}$hydraté. On sait que l'ion césium (de rayon ionique $R_{\mathrm{Cs}^{+}}=1,67 \AA$ ) est peu hydraté : 1,6 molécule d'eau par ion [13]. On peut donc penser qu'il est libre d'évoluer dans la périphérie immédiate de la micelle et susceptible de se fixer sur celle-ci. Cette interprétation semble confirmée par la mesure du degré d'association $\beta_{\mathrm{Na}}$ de l'ion sodium dans la solution micellaire. En l'absence de sel, $\beta_{\mathrm{Na}^{+}}$est voisin de 0,6 [10]; en présence d'ions $\mathrm{Cs}^{+}$(pour $\left[\mathrm{Cs}^{+}\right]=5 \times 10^{-2}$ mole. $\left.1^{-1}\right) \beta_{\mathrm{Na}^{+}}$diminue fortement $\left(\beta_{\mathrm{Na}^{+}} \simeq 0,16\right.$ [10]). Si l'on admet que le degré d'association total des contre-ions $\mathrm{Na}^{+}$et $\mathrm{Cs}^{+}$ne varie pas, on peut faire l'hypothèse qu'un certain nombre de sites occupés par $\mathrm{Na}^{+}$sont ici occupés par des ions $\mathrm{Cs}^{+}$. L'ion $\mathrm{Cs}^{+}$ hydraté étant plus petit que l'ion $\mathrm{Na}^{+}$hydraté favorise le rapprochement des têtes polaires et l'aggrégation d'un nombre plus élevé de monomères par micelle.

- La solubilisation d'ions $\mathrm{Li}^{+}$n'entraîne qu'une légère augmentation de la taille micellaire. Cet effet est cependant trop faible (pratiquement dans le domaine d'incertitude sur $n$ ) pour que l'on puisse en tirer des conclusions significatives. Il faut cependant noter que le gonflement de la micelle observé ici s'interprète difficilement par la taille de l'ion solubilisé.

\section{Conclusion.}

La possibilité de déterminer, par diffusion neutronique, le volume micellaire ainsi que le volume du cour paraffinique conduit sans ambiguịté aux dimensions de la particule pour le modèle le plus probable.

La comparaison des résultats obtenus pour les micelles de $\mathrm{C}_{8} \phi \mathrm{SO}_{3} \mathrm{Li}$ et de $\mathrm{C}_{8} \phi \mathrm{SO}_{3} \mathrm{Na}$ nous a permis de proposer un modèle sphérique pour les premières et ellipsoïdal pour les secondes. En l'absence d'interaction, deux facteurs commandent l'organisation micellaire : la longueur de la chaîne paraffinique de l'alkylbenzène et la nature du contre-ion. 


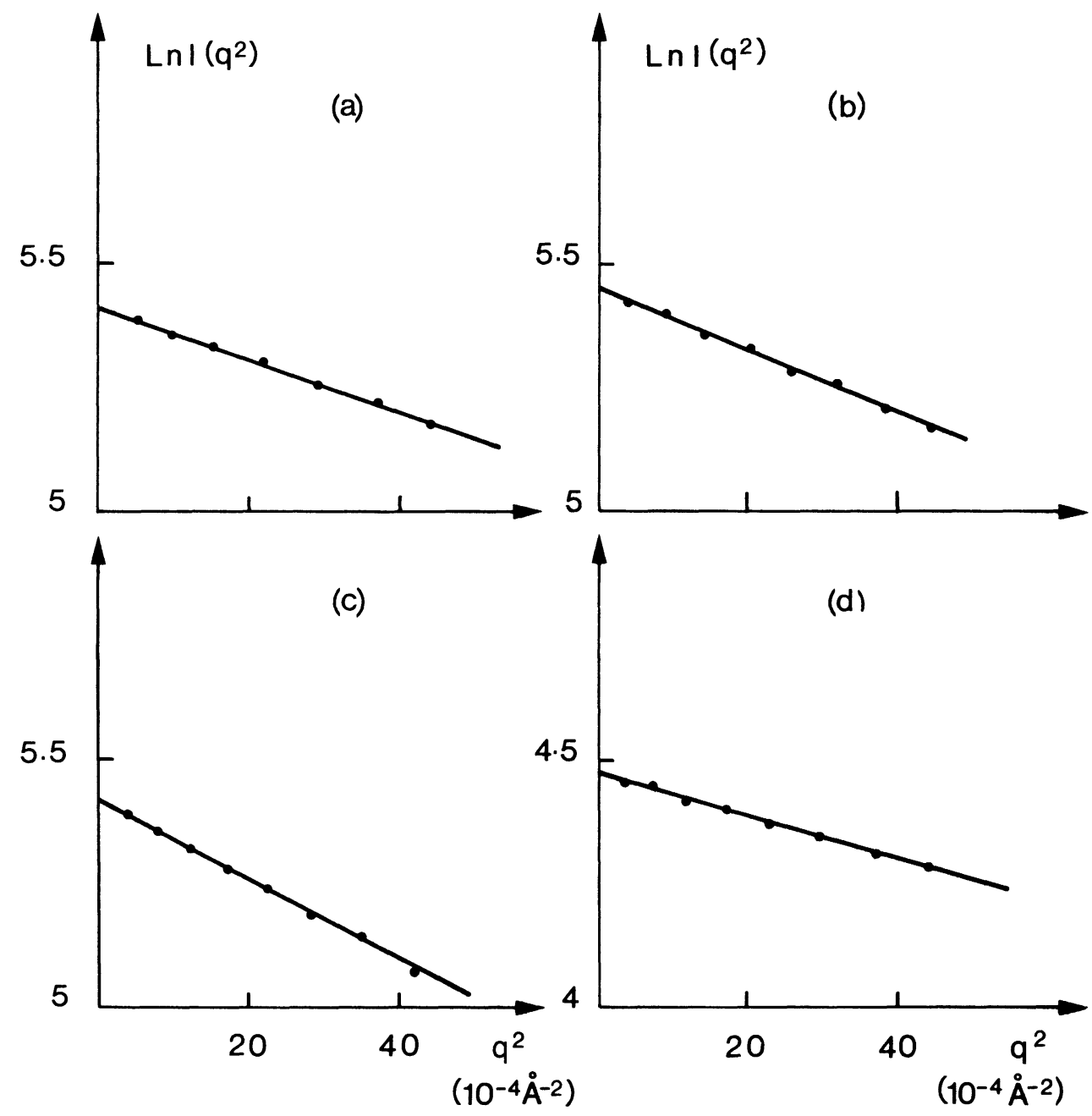

Fig. 4. - Courbes expérimentales dans la représentation de Guinier pour $\rho_{\mathrm{S}}=\rho_{\mathrm{pol}}$, [amphiphile] $=$ $=0,05$ mole. $1^{-1}[\mathrm{sel}]=0,05$ mole. $1^{-1}$. a) $\mathrm{C}_{8} \phi \mathrm{SO}_{3} \mathrm{Na}\left(R_{\mathrm{G}}\right.$ par $\left.)=12,8 \AA, b\right) \mathrm{C}_{8} \phi \mathrm{SO}_{3} \mathrm{Na}+\mathrm{LiCl}$, $\left(R_{\mathrm{G}}\right.$ par $\left.\left.)=13,5 \AA, c\right) \mathrm{C}_{8} \phi \mathrm{SO}_{3} \mathrm{Na}+\mathrm{CsCl}\left(R_{\mathrm{G}} \mathrm{par}\right)=15,5 \AA, d\right) \mathrm{C}_{8} \phi \mathrm{SO}_{3} \mathrm{Li}\left(R_{\mathrm{G}}\right.$ par $)=11,3 \AA$.

[Guinier plot of experimental curves for $\rho_{\mathrm{S}}=\rho_{\text {pol }}$, [amphiphile] $=0.05$ mole..$^{-1}$ [salt] $=0.05$ mole $.1^{-1}$. a) $\mathrm{C}_{8} \phi \mathrm{SO}_{3} \mathrm{Na}\left(R_{\mathrm{G}}\right.$ par $\left.)=12.8 \AA, b\right) \mathrm{C}_{8} \phi \mathrm{SO}_{3} \mathrm{Na}+\mathrm{LiCl}\left(R_{\mathrm{G}}\right.$ par $\left.)=13.5 \AA, c\right) \mathrm{C}_{8} \phi \mathrm{SO}_{3} \mathrm{Na}+\mathrm{CsCl}$ $\left(R_{\mathrm{G}}\right.$ par $\left.)=15.5 \AA, d\right) \mathrm{C}_{8} \phi \mathrm{SO}_{3} \mathrm{Li}\left(R_{\mathrm{G}} \mathrm{par}\right)=11.3 \AA$.]

La variation de la force ionique, par addition d'un sel, modifie suffisamment la structure micellaire pour que cet effet puisse être observé. Les modifications importantes de taille s'interprètent par l'effet de compétition entre des ions hydratés de volumes différents.

Plus généralement, dans le cas des systèmes étudiés, l'anisotropie des particules augmente lorsque leur volume croît, le rapport $v_{\text {par }} / v$ restant pratiquement constant.

Nous avons limité cette étude à deux détergents couramment employés dans l'industrie pour la récupération assistée du pétrole. On peut envisager, suivant la même technique, une étude systématique de l'effet de sel dans la phase $\mathrm{L}_{1}$ des microémulsions $\mathrm{C}_{8} \phi \mathrm{SO}_{3} \mathrm{Na}$-alcool-eau [16]. 


\section{Bibliographie}

[1] Cabos, C., Delord, P. et Rouviere, J., J. Appl. Cryst. 10 (1977) 37.

[2] Cabos, C. et Delord, P., J. Physique 39 (1978) 432.

[3] Cabos, C., Delord, P. et Martin, J. C., J. Physique Lett. 40 (1979) L-407.

[4] Cabos, C. et Delord, P., J. Appl. Cryst. 12 (1979) 502.

[5] Sardet, C., Tardieu, A. et Luzzati, V., J. Mol. Biol. 105 (1976) 383.

[6] Reiss-Husson, F. et Luzzati, V., J. Phys. Chem. 68 (1964) 3504.

[7] Guinier, A. et Fournet, G., Small Angle Scattering of X-Rays (New York John Wiley) 1955.

[8] Luzzati, V., Tardieu, A. et Mateu, L., J. Mol. Biol. 101 (1976) 115.

[9] Gray, F. W., J. Am. Chem. Soc. 61 (1939) 539.

[10] Kamenka, N., Colloid Polymer Sci. 257 (1979) 757.

[11] Shinoda, K. T., Colloidal Surfactants : Some physicochemical properties (New York) 1963.

[12] Mering, J. et Tchoubar, D., J. Appl. Cryst. 2 (1969) 128.

[13] Franks, F., Water (vol. III) 431.

[14] Gurewitch, I. I. et Tarasov, L. V., Low Energy Neutron Physics (North Holland) 1968.

[15] TANFORD, C., The hydrophobic effect : formation of micelles and biological membranes (New York, John Wiley) 1973.

[16] Larche, F., Dussossoy, J. L., Marignan, J., Rouviere, J., J. Colloid Inter. Sci. (à paraître).

[17] Zana, R., Picot, C., Duplessix, R., J. Colloid Inter. Sci. 93 (1983) 43. 